\title{
Spin-Orbital Function Formalism and ASPIRRIN Code
}

\author{
E.A.Perevedentsev*, V.Ptitsyn ${ }^{\dagger}$ and Yu.M.Shatunov* \\ ${ }^{*}$ Budker Institute for Nuclear Physics, Novosibirsk, Russia \\ ${ }^{\dagger} C-A$ Department, Brookhaven National Laboratory, Upton, NY, USA
}

\begin{abstract}
A spin-orbital function formalism is described. The formalism was realized in ASPIRRIN code that does beam polarization calculations at the first order. The code has been used for calculating equlibrium polarization and polarization time in electron rings with the complex geometry of applied magnetic fields as well for resonance strength analysis for proton accelerators.
\end{abstract}

\section{INTRODUCTION}

The ASPIRRIN (Analysis of SPIn Resonances in RINgs) is a code designed to calculate beam polarization related quantities for a charged particle beam circulating into an accelerator or storage ring. The code was written some years ago and have been used for polarization calculations for different accelerators. The paper desribes the underlying formalism used by the code, called the spin-orbital function formalism.

The code, and underlying formalism, involves polarization calculation at the first order, thus revealing effect of first-order spin resonances. Therefore results produced by the code have to agree with results obtained by other first order codes, like SLIM and SLICK [1]. The various calculations done for different accelerator lattices have demonstrated a good agreement between the ASPIRRIN and SLIM codes.

\section{SPIN-ORBITAL FUNCTIONS}

The ASPIRRIN algorithm is based on the calculation of a special set of functions, named spin-orbital functions. These functions are complex functions and connect the motion of individual particle in a circular accelerator with the motion of particle spin.

For description of the transverse and longitudinal orbital motion one can use the set of canonical variables $\left(x, p_{x}, z, p_{z}, \sigma, p_{\sigma}\right)$, which forms the orbital vector $X$. In linear approximation, the orbital 6-D dynamic is governed by the equation:

$$
X^{\prime}=S H X+Q
$$

where $H$ is symmetrical hamiltonian matrix and $S$ is the fundamental symplectic matrix. The vector $Q$ includes effects from bending field errors.

The description of spin motion in circular accelerators are based on two important quantities, the periodical spin field $\hat{\mathbf{n}}$ and the spin tune $v_{s}$. 
The periodicity of the $\hat{\mathbf{n}}$ can be expressed as:

$$
\hat{\mathbf{n}}\left(x, p_{x}, z, p_{z}, \sigma, p_{\sigma}, \theta+2 \pi\right)=\hat{\mathbf{n}}\left(x, p_{x}, z, p_{z}, \sigma, p_{\sigma}, \theta\right)
$$

which means that at the given azimuth $\theta$ the $\hat{\mathbf{n}}$ is function of orbital phase space point. The large variations of $\hat{\mathbf{n}}$ over orbital motion phase space in spin resonance zones lead to possible beam depolarization during the resonance crossing. The spin-orbital function formalism aims at the spin field $\hat{\mathbf{n}}$ calculation, taking first-order approximation both in orbital and spin motion. Having it calculated, the analysis can be done for depolarization effects.

The suitable reference system to do the spin calculations is the system formed by $\hat{\mathbf{n}}_{0}, \eta_{1}, \eta_{2}$ unit vectors [2]. $\hat{\mathbf{n}}_{\mathbf{0}}$ is the periodical spin solution on the reference beam orbit in an accelerator (without magnet errors and mislaignments). $\hat{\eta}_{1}$ and $\hat{\eta}_{2}$ vectors are spin solutions on the reference orbit, that are orthogonal to $\hat{\mathbf{n}}_{\mathbf{0}}$ and to each other. In this reference frame any spin solution, including the vector $\hat{\mathbf{n}}$, can be described by a complex variable $C$ as:

$$
\hat{\mathbf{n}}=\sqrt{1-|C|^{2}} \hat{\mathbf{n}}_{\mathbf{0}}+\operatorname{Re}\left(i C \hat{\eta}^{*}\right)
$$

where $\hat{\eta}=\hat{\eta}_{1}-i \hat{\eta}_{2}$. The differential equation for $C$ variable can be derived from the equation of spin rotation in an external field. In the first-order approximation the equation for $C$ simplifies to:

$$
C^{\prime}=w_{\perp}
$$

with $w_{\perp}=\mathbf{w} \hat{\eta}$, where the spin precession vector $\mathbf{w}$ describes the precession due to betatron and synchrotron oscillations and field errors:

$$
\begin{aligned}
& w_{x}=\left(1+v_{0}\right) z^{\prime \prime}+\left(v_{0}+\frac{a}{\gamma_{0}}\right) K_{x} p_{\sigma}+(1+a) K_{y} x^{\prime} \\
& w_{y}=(1+a)\left(K_{x}^{\prime} x+K_{z}^{\prime} z+\Delta K_{y}-K_{y} p_{\sigma}\right)-\left(v_{0}-a\right)\left(K_{x} p_{x}+K_{z} p_{z}\right) \\
& w_{z}=-\left(1+v_{0}\right) x^{\prime \prime}+\left(v_{0}+\frac{a}{\gamma_{0}}\right) K_{z} p_{\sigma}+(1+a) K_{y} z^{\prime}
\end{aligned}
$$

Since, according to (2), $\hat{\mathbf{n}}$ is the function of the orbital phase space point, it can be expanded in a power set of orbit variables. In the first-order approximation, one should leave just the linear part of the expansion, which we present in the form:

$$
C=\left(1+v_{0}\right)\left(z^{\prime} \eta_{x}-x^{\prime} \eta_{z}\right)+f_{0}+F^{T} S X
$$

where $F$ is 6-D vector, $v_{0}=\gamma a$ and $a$ is the anomalous magnetic moment of the particle. The components of this vector, $F_{i}(i=1 . .6)$ are functions of the ring azimuth $\theta$. Together with the scalar function $f_{0}(\theta)$ they form the set of functions which we call spin-orbital functions.

To derive the equation which describes the spin-orbital function evolution along $\theta$ one can substitute the expression (5) into the spin equation (4). After some transformations it leads to following equations:

$$
\begin{aligned}
F^{\prime} & =S H F+P \\
f_{0}^{\prime} & =\Delta K_{z} F_{1}-\Delta K_{x} F_{3}+(1+a) \Delta K_{y} \eta_{y}
\end{aligned}
$$


The function $f_{0}$ is generated by field errors $\Delta K_{i}$ on the reference orbit.

The equation for the vector $F$ is quite similar to the equation (1) governing the orbital motion. The linear parts of the equations (6) and (1) are exactly the same. The components of vector $P$ are present only in dipole or solenoidal magnets:

$$
\begin{array}{ll}
P_{1}=-\left(v_{0}-a\right) K_{y} \eta_{x}+\left(v_{0}^{2}+a\right) K_{x} \eta_{y} ; & P_{2}=-(1+a) K_{x}^{\prime} \eta_{y}-\left(v_{0}-a\right) \frac{1}{2} K_{y}^{2} \eta_{z} \\
P_{3}=\quad\left(v_{0}^{2}+a\right) K_{z} \eta_{y}-\left(v_{0}-a\right) K_{y} \eta_{z} ; & P_{4}=\left(v_{0}-a\right) \frac{1}{2} K_{y}^{2} \eta_{x}-(1+a) K_{z}^{\prime} \eta_{y} \\
\left.P_{5}=\left(v_{0}+\frac{a}{\gamma_{0}}\right)\left(K_{x} \eta_{x}+K_{z} \eta_{z}\right)-1+a\right) K_{y} \eta_{y} ; & P_{6}=0
\end{array}
$$

where $K_{i}=B_{i} /\left\langle B_{z}\right\rangle$ describes normalized magnetic field.

The similarity of linear terms of the equations of (6) and (1) leads to the important conclusion. The well-known transfer matrices for accelerator elements, routinely used for calculation of the linear orbital motion, can be applied for the calculation of spinorbital functions too. The transformation of vector $F$ trough an accelerator element can be written as:

$$
F_{\text {out }}=M F_{\text {in }}+Y
$$

where $M$ is the transfer matrix for given element and the transfer vector $Y$, generated by the vector $P$, exists at the bending and solenoidal magnets. Making element-by-element transformation one comes to the one turn transformation in the form:

$$
F(2 \pi)=M_{r e v} F(0)+Y_{\text {rev }}
$$

The periodicity conditions for the vector $\hat{\mathbf{n}}$ lead to the following conditions for spinorbital functions:

$$
F_{i}(\theta+2 \pi)=e^{i 2 \pi v} F_{i}(\theta), \quad f_{0}(\theta+2 \pi)=e^{i 2 \pi v} f_{0}(\theta)
$$

Then, from expressions (9) and (10) one can find the solution for vector $F$ at $\theta=0$

$$
F(0)=\left(I \cdot \mathrm{e}^{i 2 \pi v}-M_{\text {rev }}\right)^{-1} Y_{\text {rev }}
$$

The value of vector $F$ at any other element of a ring can be found then by doing again element-by-element transformation (8). Because eigen values of $M_{\text {rev }}$ matrix are $\mathrm{e}^{i 2 \pi v_{i}}$, where $v_{i}$ are tunes of the orbital motion, the resonance denominator in (11) shows the first order spin resonances when $v=m \pm v_{i}$.

Another consequence of the linear parts identity of (6) and (1) is that the spin-orbital functions can be related through integral transformations to characteristic functions of the orbital motion ( $\beta$-functions and orbital motion phases).

In an accelerator with only vertical guiding field on the particle reference orbit and without field errors the spin motion is coupled only with vertical orbital motion, thus only $F_{3}$ and $F_{4}$ functions have not zero values. An introduction of horizontal or solenoidal fields, for example as a part of a spin rotator insertion, leads to exciting a whole set of the spin-orbital functions. 

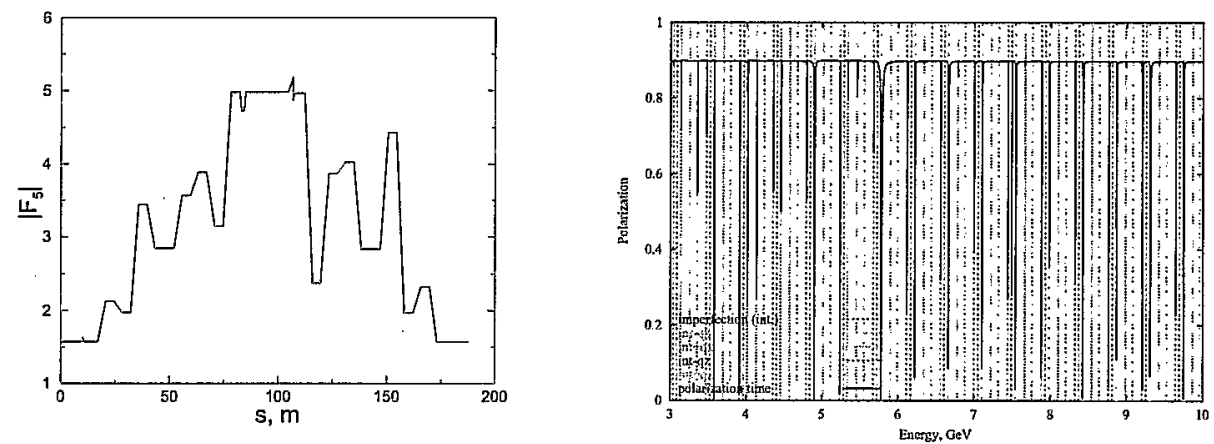

FIGURE 1. The examples of the ASPIRRIN calculations. On the left plot: $\left|F_{5}\right|$ for the Bates SHR with Siberian snake and wigglers. On the right plot: equilibrium polarization at proposed EIC electron ring.

\section{APPLICATION OF THE SPIN-ORBITAL FUNCTIONS}

The set of functions $F$ can be used for calculating of various polarization characteristics of polarized beams in accelerators.

\section{Calculation of equilibrium polarization and depolarization time}

For electron rings, very important quantity is the partial derivative of $\hat{\mathbf{n}}$ over longitudinal momentum variable, $\mathbf{d}=\partial \hat{\mathbf{n}} / \partial \mathbf{p}_{\sigma}$, which defines the equilibrium polarization and depolarization time due to synchrotron radiation process. In the spin-orbital function formalism the vector $\mathbf{d}$ is connected with $F_{5}$ component of the vector $F: \mathbf{d}=\operatorname{Re}\left(i F_{5} \hat{\eta}^{*}\right)$

Let us note that: $|\mathbf{d}|=\left|F_{5}\right|$ and that $\left|F_{5}\right|$ is periodical function of $\theta$. Thus, with $F_{5}$ calculated one can calculate also the equilibrium polarization and depolarization time according to Derbenev-Kondratenko formula [2].

Some examples of the polarization calculation by ASPIRRIN code are shown in Figure 1. Left figure demonstrates the calculated $\left|F_{5}\right|$ for South Hall Ring at MITBates with the solenoidal Siberian snake and polarizing wigglers. On the right plot the polarization degree is shown for the electron ring of electron-ion collider project [3], with the spin depolarizing resonance pattern present.

Like $F_{5}$ function (or $|\mathbf{d}|$ vector) is used to calculate depolarizing effect of spin diffusion caused by sudden particle energy changes due to synchrotron radiation, the $F_{3}$ and $F_{1}$ spin-orbital function can be applied to calculate the spin diffusion caused by particle transverse momentum changes in scattering processes.

\section{Calculation of resonance strength}

Another possible application of spin-orbital functions is for the spin resonance strength analysis. We show this on the example of calculation of imperfection resonance 
strength.

As seen from (7) the $f_{0}$ function is generated by the magnetic field errors. Therefore this function should describe imperfection resonances. Indeed, the solution for $f_{0}$ is:

$$
f_{0}=\frac{1}{\mathrm{e}^{i 2 \pi v}-1} \int_{\theta}^{\theta+2 \pi}\left(\Delta K_{z} F_{1}-\Delta K_{x} F_{3}+(1+a) \Delta K_{y} \eta_{y}\right) d \theta
$$

And for an imperfection resonance strength one can get:

$$
\left|w_{k}\right|=\frac{1}{2 \pi}\left|\int_{0}^{2 \pi} f_{0(v=m)}^{\prime} d \theta\right|=\frac{1}{2 \pi}\left|\int_{0}^{2 \pi}\left(\Delta K_{z} F_{1}-\Delta K_{x} F_{3}+(1+a) \Delta K_{y} \eta_{y}\right) d \theta\right|
$$

where $F_{1}, F_{3}$ and $\eta_{y}$ are calculated at the resonance condition $v=m$. Thus, the contributions to the resonance strength from horizontal, vertical and solenoidal field errors are described by the functions $F_{3}, F_{1}$ and $\eta_{y}$ respectively. Let us note that in this approach it is not required to calculate the contribution to the resonance strength coming from closed orbit distortions produced by the field errors. It is taken into account automatically.

The similar approach can be used to calculate the resonance strength generated by coupling or gradient errors in an accelerator [4].

\section{CONCLUSION}

The set of spin-orbital function $F$ was introduced and was shown to be useful for polarization calculation for particle beams in circular accelerators with complex magnet field configuration. The spin-orbital function formalism was put in the base of the ASPIRRIN code, which calculates the equilibrium polarization and depolarization time for electron rings as well as strength of spin resonances.

\section{ACKNOWLEDGMENTS}

We would like to thank D. Barber (DESY), I. Koop, A. Otboev (BINP) and F. Wang (MIT) for interesting discussions and valuable comments concerning the ASPIRRIN code. U.S. Department âf Energy.

\section{REFERENCES}

1. A.W.Chao, NIM 180, p.29(1981).

D.P. Barber, Private notes, (1982).

2. Ya. S. Derbenev, A. M. Kondratenko, Sov. Phys. JETP, 37, p.968 (1973).

3. Yu.M. Shatunov, et al, "Status of the e-ring design for EIC", these proceedings.

4. V. Ptitsyn, Ph. D. Theses, p.26 (1997). 